\title{
Frequency-domain Identification of the Human Controller
}

\author{
Henrik Gollee · Adamantia Mamma · Ian D \\ Loram · Peter J Gawthrop
}

the date of receipt and acceptance should be inserted later

\begin{abstract}
System identification techniques applied to experimental human-in-the-loop data provide an objective test of three alternative control-theoretical models of the human control system: non-predictive control, predictive control, and intermittent predictive control. A two-stage approach to the identification of a single-input single-output control system is used: first, the closed-loop frequency response is derived using the periodic property of the experimental data, followed by the fitting of a parametric model. While this approach is well-established for non-predictive and predictive control, it is here used for the first time with intermittent predictive control. This technique is applied to data from experiments with human volunteers who use one of two control strategies, focusing either on position or on velocity, to manually control a virtual, unstable load which requires sustained feedback to maintain position or low velocity. The results show firstly that the non-predictive controller does not fit the data as well as the other two models, and secondly that the predictive and intermittent predictive controllers provide equally good models which cannot be distinguished using this approach. Importantly, the second observation implies that sustained visual manual control is compatible with intermittent control, and that previous results suggesting a continuous control model for the human control system do not rule out intermittent control
\end{abstract}

H. Gollee · A. Mamma · P. J. Gawthrop

School of Engineering, University of Glasgow, Glasgow, UK. E-mail: henrik.gollee@glasgow.ac.uk

\section{D. Loram}

Institute for Biomedical Research into Human Movement and Health, Manchester Metropolitan University, Manchester, UK 
as an alternative hypothesis. Thirdly, the parameters identified reflect the control strategy adopted by the human controller.

Keywords Intermittent control; predictive control; optimal control; human operator; human balancing.

\section{Introduction}

Physiological control systems have been the subject of research over an extended period. One topic of debate is whether human motor control mechanisms can be modelled as technological control systems and, if so, what control algorithm is used.

One thread of research involves using the proportional-integral-derivative (PID) control algorithm - a long-standing process control algorithm. This approach was suggested some time ago (Johansson et al. 1988) and has received a lot of attention recently (Peterka 2002; Maurer and Peterka 2005; Alexandrov et al. 2005; Masani et al. 2006; Lockhart and Ting 2007; van der Kooij and de Vlugt 2007; Welch and Ting 2008; van der Kooij and Peterka 2011). Typically, the three gain parameters and time delay of the PID model have been tuned to simulate spontaneous sway (Maurer and Peterka 2005) or have been tuned to reproduce the stimulus-response data where the support surface has been pseudo-randomly rotated (Peterka 2002) or translated (Alexandrov et al. 2005; Welch and Ting 2008). This simple delayed-feedback model has been shown many times to fit the data quite well; and, in the absence of any contradictory evidence, support remains for the idea that predictive processes are not required to explain standing balance.

Another thread of research in this area involves modelling the human operator as a predictive feedback control system (Kleinman 1969; van der Kooij et al. 1999). In particular, the continuous-time state-observer, state-predictor, state-feedback control structure of Kleinman (1969) provides a model of human control systems which has been shown to be applicable under a range of experimental conditions. Kleinman et al. (1970) and Baron et al. (1970) showed that this model explained many features of the human operator performing compensatory tracking including the general features of the error signals - called the remnant in this context (Levison et al. 1969). In his survey paper, McRuer (1980) states that this "algorithmic model" of the human operator "works well for imitating human behaviour".

Both the non-predictive and predictive approach are continuous-time algorithms. In contrast, a third thread of research starting with Craik (1947a,b) and Vince (1948) and further 
developed by Navas and Stark (1968) and Neilson et al. (1988) suggests an intermittent basis for human control systems. The first algorithmic intermittent control model was presented by Neilson et al. (1988) and expanded in subsequent papers including Neilson and Neilson (1999); Neilson (1999); Neilson and Neilson (2005); Oytam et al. (2005); Bye and Neilson (2008); Gawthrop et al. (2011). In the control-engineering literature, intermittent control has been developed in a series of papers initiated by Ronco et al. (1999) and continuing with Gawthrop (2002, 2004) and Gawthrop and Wang (2006, 2007); In (Gawthrop 2009), frequency domain properties of intermittent control have been derived.

There are thus three competing hypotheses: non-predictive control, predictive control and intermittent predictive control. These competing hypotheses must be tested using experimental data. System identification provides one approach to hypothesis testing and has been used by Johansson et al. (1988) and Peterka (2002) to test the non-predictive hypothesis and by Gawthrop et al. (2009) to test the non-predictive and predictive hypotheses. Peterka (2002) uses a two stage approach to controller estimation: firstly, the transfer function frequency response is estimated and secondly a parametric model is fitted to the frequency response using non-linear optimisation. Such a two stage approach which has also been suggested by Pintelon and Schoukens (2001); Pintelon et al. (2008) and is well-established in the engineering literature, is adopted here. In particular, the advantageous properties of a periodic input signal (as advocated by Pintelon et al. (2008)) are used to estimate the frequency response which is then fitted by a parametric model based on each of the three competing hypotheses.

Identification of physiological control systems from unperturbed measured data has two problems: first, the controller is embedded in a closed-loop system and second, the need to estimate disturbance models. Both can lead to ambiguity in the interpretation of the results, and these two pitfalls are avoided here by using an external measured perturbation to the system and by identifying the entire closed-loop dynamics (van der Kooij et al. 2005).

An earlier paper (Gawthrop et al. 2009) used time-domain identification and random non-periodic signals to compare the predictive and non-predictive hypotheses and found that the predictive controller gave a better fit to the data taking account of the estimated timedelay. In the present paper a frequency domain approach with a periodic excitation signal is used instead, and a third hypothesis - intermittent predictive control - is investigated in this context for the first time. 
Section 2 describes the three hypothesised controllers and Section 3 gives the corresponding closed-loop frequency responses. Section 4 describes the two-stage identification procedure and Section 5 applies the procedure to simulated data to verify the properties of the method. Section 6 describes the experimental results and Section 7 concludes the paper.

\section{System and Controller Models}

Dynamical systems can be represented in either state-space or transfer function form. The choice of representation is not a fundamental issue but rather a matter of convenience: either representation can be converted into the other. This paper uses a state-space approach to directly make use of the state-space predictor formulation of Kleinman (1969) and because it is the natural setting for intermittent control.

As indicated in Figure 1, this paper considers single-input single-output time-delay systems with an input disturbance given in state-space form by

$$
\begin{aligned}
\dot{x}(t) & =A x(t)+B[u(t)-d(t)] \\
y(t) & =C x(t) \\
u(t) & =u_{0}(t-\Delta)
\end{aligned}
$$

where $\Delta$ is the time-delay and $d(t)$ an additive disturbance. Three candidate controllers are considered: a non-predictive controller (Figure 1(a)), a predictive controller (Figure 1(b)) and an intermittent predictive controller controller (Figure 1(c)).

2.1 Non-predictive control (NPC)

The standard non-predictive state-space control equations are:

$$
\begin{array}{rlrl}
\dot{\hat{x}}(t) & =A \hat{x}(t)+B u_{0}(t)-L[C \hat{x}(t)-y(t)] & \\
& =A_{o} \hat{x}(t)+B u_{0}(t)+L y(t) & & \text { (Observer) } \\
u_{0}(t) & =-k \hat{x}(t) & & \text { (Controller) }
\end{array}
$$

where the observer matrix $A_{o}$ is given by:

$$
A_{o}=A-L C
$$

where $L$ is the observer gain. 
(a) Non-predictive control

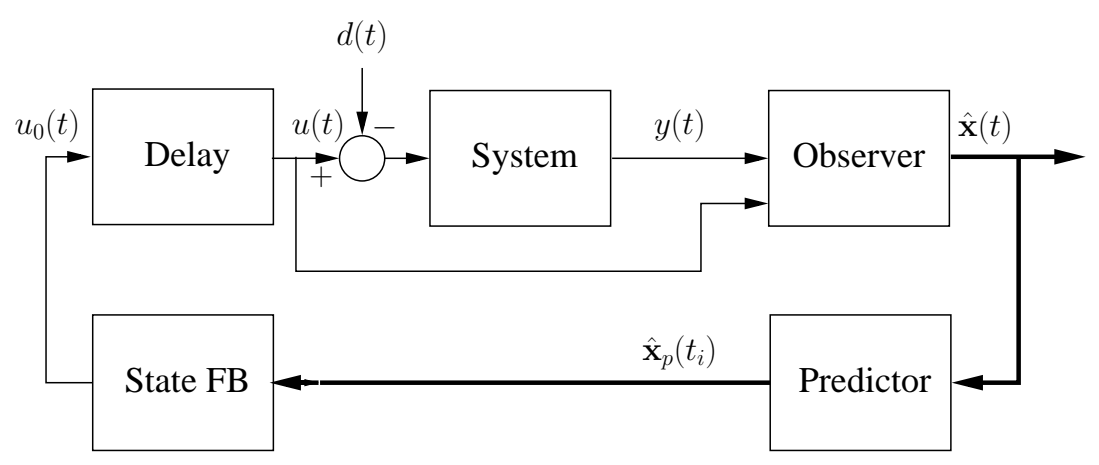

(b) Predictive control 
There are many ways of choosing the feedback gain $k$. It is a standard result that, in the absence of a time delay, the closed loop system poles are the eigenvalues of $A_{o}(6)$ together with the eigenvalues of $A_{c}$ where:

$$
A_{c}=A-B k
$$

Unlike the predictive controller of Section 2.2 and the intermittent predictive controller (Section 2.3), this controller does not take explicit account of the system time delay.

2.2 Predictive control (PC)

Following Kleinman (1969), the predictive controller equations corresponding to the timedelay system of Equations (1) and (2), can be written as:

$$
\begin{aligned}
\dot{\hat{x}}(t) & =A \hat{x}(t)+B u(t)-L[C \hat{x}(t)-y(t)] & & \\
& =A_{o} \hat{x}(t)+B u(t)+L y(t) & & \text { (Observer) } \\
\hat{x}_{p}(t) & =e^{A \Delta} \hat{x}(t)+\int_{0}^{\Delta} e^{A \tau} B u_{0}(t-\tau) d \tau & & \text { (Predictor) } \\
u_{0}(t) & =-k \hat{x}_{p}(t) & & \text { (Controller) }
\end{aligned}
$$

where $\hat{x}_{p}(t)$ is the prediction of $\hat{x}(t+\Delta)$ at time $t$ and the state estimate $\hat{x}(t)$ is given by the observer equation (8). Unlike the observer of equation (4), equation (8) uses the delayed control $u(t)=u_{0}(t-\Delta)$.

\subsection{Intermittent Control (IC)}

The intermittent predictive controller of Figure 1(c) is similar to the predictive controller of Figure 1(b) in that it shares the same observer, predictor and state feedback. However, it differs from the predictive controller of Figure 1(b) in three ways:

1. The observer state $\hat{x}$ is sampled.

2. There is a vector generalised hold element to reconstruct the sampled signal.

3. The predictor operates on a sampled signal.

Item 3 leads to a much simpler formula than that for the continuous case given by equation (9); details are given by (Gawthrop and Wang 2007, Section 4.2.). The key issue for this paper is the design of the hold element of item 2 which is now discussed.

Intermittent control makes use of three time frames: 
1. continuous-time, within which the controlled system (1) and (2) evolves, denoted by $t$.

2. discrete-time points at which feedback occurs indexed by $i$. Thus, for example, the discrete-time instants are denoted $t_{i}$ and the corresponding estimated state is $\hat{x}_{i}=\hat{x}\left(t_{i}\right)$.

The $i$ th intermittent interval is defined as

$$
\Delta_{i}=t_{i+1}-t_{i}
$$

In this paper we assume that all intermittent intervals have the same duration, denoted as $\Delta_{o l}$.

3. intermittent-time is a continuous-time variable, denoted by $\tau$, restarting at each intermittent interval. Thus, within the $i$ th intermittent interval:

$$
\tau=t-t_{i}
$$

In particular, the control signal $u(t)=u\left(t_{i}+\tau\right)$ for $t_{i} \leq t<t_{i+1}$.

This paper uses a special case of the generalised hold which has a simple intuitive interpretation. In particular, the generalised hold here is a disturbance-free open-loop simulation of the actual closed-loop system which is reinitialised to the estimated system state $\hat{x}$ at each sample interval $t_{i}$. The hold state $\hat{x}_{h}$ evolves in the intermittent time frame $\tau$ as

$$
\begin{cases}\frac{d}{d \tau} \hat{x}_{h}(\tau) & =A_{c} \hat{x}_{h}(\tau) \\ \hat{x}_{h}(0) & =\hat{x}_{p}\left(t_{i}-t_{d}\right)\end{cases}
$$

where $A_{c}$ is the closed-loop system matrix (7). As discussed elsewhere (Gawthrop and Wang 2007; Gawthrop 2009), this particular choice of hold means that the low-frequency behaviour of the intermittent controller is similar to that of the predictive controller.

One effect of the hold operation is that the loop time-delay with respect to some discrete event is not constant: depending on when the intermittent interval is restarted, the timedelay $\Delta$ may be increased by between 0 and $\Delta_{o l}$. On average, the effective loop time delay is therefore

$$
\Delta_{e}=\Delta+0.5 \Delta_{o l} .
$$




\section{Closed-loop Frequency responses}

The system equations can be rewritten in transfer function form ${ }^{1}$ as:

$$
\begin{aligned}
y(s) & =G(s) u(s) \\
& =e^{-s \Delta} G(s) u_{0}(s) \\
\text { where } G(s) & =C[s I-A]^{-1} B
\end{aligned}
$$

where $I$ is the $n \times n$ unit matrix.

As explicitly derived in Sections 3.1 and 3.2, the first two controllers have transfer function representations $H(s)$ where:

$$
\frac{u_{0}(s)}{y(s)}=-H(s)
$$

In this case it follows that the system loop-gain $L(s)$ and closed-loop transfer function $T$ are given by:

$$
\begin{aligned}
L(s) & =e^{-s \Delta} G(s) H(s) \\
T(s) & =\frac{L(s)}{1+L(s)} \\
\text { with } \quad \frac{u(s)}{d(s)} & =T(s)
\end{aligned}
$$

The next two sections give expressions for $H(s)$ for the predictive and non-predictive cases and thus, using (18) and (20) give a parametrised expression for the transfer function $T(s)$ relating $u(s)$ and $d(s)$.

\subsection{Non-predictive control}

Transforming equations (4)-(5) into the Laplace domain:

$$
\begin{aligned}
\hat{x}(s) & =\left(s I-A_{o}\right)^{-1}\left(B u_{0}(s)+L y(s)\right) & & \text { (Observer) } \\
u_{0}(s) & =-k \hat{x}(s) & & \text { (Controller) }
\end{aligned}
$$

Equations (21)-(22) can be rewritten as:

$$
\begin{aligned}
u_{0}(s) & =-H(s) y(s) \\
\text { where } H(s) & =k\left(s I-A_{o}+B k\right)^{-1} L
\end{aligned}
$$

\footnotetext{
1 To avoid proliferation of notation, symbols followed by (s) are taken to be the the Laplace transform of the quantity indicated by the same symbol but followed by (t)
} 
3.2 Predictive control

Transforming equations (8)-(10) into the Laplace domain:

$$
\begin{array}{rr}
\hat{x}(s)=\left(s I-A_{o}\right)^{-1}\left(e^{-s \Delta} B u_{0}(s)+L y(s)\right) & \text { (Observer) } \\
\hat{x}_{p}(s)=e^{A \Delta} \hat{x}(s)+(s I-A)^{-1}\left(I-e^{-(s I-A) \Delta}\right) B u_{0}(s) \\
u_{0}(s)=-k \hat{x}_{p}(s) & \text { (Predictor) } \\
& \text { (Controller) }
\end{array}
$$

where $I$ is the $n \times n$ unit matrix.

Equations (25)-(27) can be rewritten as:

$$
\begin{aligned}
u_{0}(s) & =-H_{y}(s) y(s)-\left(H_{1}(s)+H_{2}(s)\right) u_{0}(s) \\
\text { where } H_{y}(s) & =k e^{A \Delta}\left(s I-A_{o}\right)^{-1} L \\
H_{1}(s) & =k e^{A \Delta}\left(s I-A_{o}\right)^{-1} B e^{-s \Delta} \\
\text { and } H_{2}(s) & =k(s I-A)^{-1}\left(I-e^{-(s I-A) \Delta}\right) B
\end{aligned}
$$

It follows that the controller transfer function $H(s)$ is given by:

$$
H(s)=\frac{H_{y}(s)}{1+H_{1}(s)+H_{2}(s)}
$$

\subsection{Intermittent Control}

The sampling operation in Figure 1(c) makes it harder to derive a (continuous-time) frequency response and so the details are omitted here. Instead, the basic result derived by Gawthrop (2009) is encapsulated as the following theorem:

Theorem 1 The continuous-time system (1) controlled by an intermittent controller with generalised hold gives a closed-loop system where the Fourier transform $U(j \omega)$ of the control signal $u(t)$ is given in terms of the Fourier transform $\mathbf{X}^{\mathbf{d}}(j \omega)$ by

$$
U(j \omega)=F(j \omega, \theta)\left[\mathbf{X}^{\mathbf{d}}(j \omega)\right]^{s}
$$


where

$$
\begin{aligned}
F(j \omega, \theta) & =\mathbf{H}(j \omega) \mathbf{S}_{\mathbf{z}}\left(e^{j \omega}\right) \\
\mathbf{H}(j \omega) & =\frac{1}{\Delta_{o l}} k\left[j \omega I-A_{c}\right]^{-1}\left[I-e^{-\left(j \omega I-A_{c}\right) \Delta_{o l}}\right] \\
\mathbf{S}_{\mathbf{z}}\left(e^{j \omega}\right) & =\left[I+\mathbf{G}_{\mathbf{z}}\left(e^{j \omega}\right)\right]^{-1} \\
\mathbf{G}_{\mathbf{z}}\left(e^{j \omega}\right) & =\left[e^{j \omega} I-A_{x}\right]^{-1} B_{x} \\
\mathbf{X}^{\mathbf{d}}(j \omega) & =\mathbf{G}(j \omega) \mathbf{d}(j \omega) \\
\mathbf{G}(j \omega) & =[j \omega I-A]^{-1} B
\end{aligned}
$$

The sampling operator is defined as

$$
\left[\mathbf{X}^{\mathbf{d}}(j \omega)\right]^{s}=\sum_{k=-\infty}^{\infty} \mathbf{X}(\mathbf{j} \omega)^{\mathbf{d}}\left(j \omega-k j \omega_{o l}\right)
$$

where the intermittent sampling-frequency is given by $\omega_{o l}=2 \pi / \Delta_{o l}$.

Proof This is a simplified version of (Gawthrop 2009, Theorem 1) for the special case of this paper.

As discussed elsewhere (Gawthrop 2009), the presence of the sampling operator $\left[\mathbf{X}^{\mathbf{d}}(j \omega)\right]^{s}$ means that the interpretation of $F(j \omega, \theta)$ is not quite the same as that of the closed loop transfer function $T(s)$ of (20), as the sample process generates an infinite number of frequencies which can lead to aliasing. As shown in Gawthrop (2009), the (bandwidth limited) observer acts as an anti-aliasing filter, which limits the effect of $\left[\mathbf{X}^{\mathbf{d}}(j \omega)\right]^{s}$ to higher frequencies and makes $F(j \omega, \theta)$ a valid approximation of $U(j \omega) . F(j \omega, \theta)$ will therefore be treated as equivalent to $T(j \omega)$ in the rest of this paper.

\section{System identification}

The aim of the identification procedure is to derive an estimate for the closed-loop transfer function of the system. Our approach follows the two stage procedure of Pintelon and Schoukens (2001) and Pintelon et al. (2008). In the first step, the frequency response transfer function is estimated based on measured input-output data, resulting in a non-parametric estimate. In the second step, a parametric model of the system is fitted to the estimated frequency response using an optimisation procedure. 
4.1 Non-parametric estimation

The procedure described in this section aims to derive a non-parametric estimate of the closed loop frequency response function (FRF), based on observed input-output data. The approach here follows that described by Pintelon and Schoukens (2001), who suggest exciting the system using a periodic multisine input signal,

$$
d(t)=\sum_{k=1}^{N_{f}} a_{k} \cos \left(\omega_{k} t+\phi_{k}\right) \quad \text { with } \omega_{k}=2 \pi k f_{0}
$$

consisting of $N_{f}$ discrete frequencies $\omega_{k}$, with resolution $\omega_{0}=2 \pi f_{0}$. The amplitude and phase of the $k t h$ frequency component are given by $a_{k}$ and $\phi_{k}$, repectively. The signal $d(t)$ is periodic with a period of $T_{0}=1 / f_{0}$. To obtain an unpredictable excitation, the phases $\phi_{k}$ are random values taken from a uniform distribution on the open interval $(0,2 \pi)$, while $a_{k}=1$ for all $k$ to ensure that all frequency are equally excited. The output $u(t)$ of a linear system which is excited by $d(t)$ then only contains information at the same discrete frequencies $\omega_{k}$ as the input signal. If the system is non-linear or noise is added, the output will contain a remnant component at non-excited frequencies. To obtain a reliable estimate of the linear system response, an average over several periods can be used, as described below.

If the signals are sampled with a sample period $T_{S}$, then the continuous time $t$ is replaced by a discrete sample time $t_{n}=n T_{s}$. A time domain signal $d\left(t_{n}\right)$ over one period $T_{0}$ can be transformed into the frequency domain using the discrete Fourier transformation (DFT),

$$
d_{D F T}\left(j \omega_{k}\right)=\sum_{n=0}^{N-1} d\left(t_{n}\right) e^{-j 2 \pi n \frac{k}{N}}, \quad k=1,2, \ldots N_{f}
$$

where $N=T_{0} / T_{s}$ is the number of sample points within one signal period, and $\omega_{k}$ is the discrete frequency with the resolution $f_{0}$. If the input signal has been applied over $N_{p}$ periods of the excitation signal, then the DFT signals for the $l$ th period can be denoted as $d^{[l]}\left(j \omega_{k}\right)$ and $u^{[l]}\left(j \omega_{k}\right)$, respectively. The frequency response transfer function for this period can be estimated as

$$
\hat{T}^{[l]}\left(j \omega_{k}\right)=\frac{u^{[l]}\left(j \omega_{k}\right)}{d^{[l]}\left(j \omega_{k}\right)}, \quad k=1,2, \ldots, N_{f}
$$

An estimate of the FRF over all $N_{p}$ periods is obtained by taking the mean,

$$
\hat{T}\left(j \omega_{k}\right)=\frac{1}{N_{p}} \sum_{l=1}^{N_{p}} \hat{T}^{[l]}\left(j \omega_{k}\right), \quad k=1,2, \ldots, N_{f}
$$


This approach ensures that only the periodic features related to the disturbance signal are used in the identification, and that the identification is robust with respect to remnant components (resulting from nonlinearities and noise). In addition to obtaining the FRF, this approach also allows to directly estimate the coherence (Pintelon and Schoukens 2001),

$$
\hat{C}_{u d}\left(j \omega_{k}\right)=\frac{\left|\sum_{l=1}^{N_{p}} u^{[l]}\left(j \omega_{k}\right) d^{[l]}\left(j \omega_{k}\right)\right|^{2}}{\sum_{l=1}^{N_{p}}\left|d^{[l]}\left(j \omega_{k}\right)\right|^{2} \sum_{l=1}^{P}\left|u^{[l]}\left(j \omega_{k}\right)\right|^{2}}
$$

With a periodic excitation, the coherence is unity in the absence of noise and becomes smaller as noise increases.

\subsection{Parametric optimisation}

In the second stage of the identification procedure, a parametric description, $\tilde{T}\left(j \omega_{k}, \theta\right)$, is fitted to the estimated FRF of equation (44). The parametric FRF approximates the closed loop transfer function (equation (20)) which depends in the case of non-predictive and predictive control, on the loop transfer function $L\left(j \omega_{k}, \theta\right)$, equation (18), parametrised by the vector $\theta$, while for the intermittent controller this is approximated by $F(j \omega, \theta)$, equation (33),

$$
\tilde{T}\left(j \omega_{k}, \theta\right)= \begin{cases}\frac{L\left(j \omega_{k}, \theta\right)}{1+L\left(j \omega_{k}, \theta\right)} & \text { for NPC and PC } \\ F\left(j \omega_{k}, \theta\right) & \text { for IC }\end{cases}
$$

A straightforward approach would be to parametrise the controller directly in terms of its feedback and observer gain vectors, $k$ and $L$ (see section 2), together with the time delay $\Delta$ and, in the case of the intermittent controller, the intermittency interval $\Delta_{o l}$. This approach has two problems: firstly it results in a closed loop system for which stability is not explicitly known, and secondly there are an excessive number of parameters. We therefore use an indirect approach were the controller and observer gains are derived using the standard LQR approach (Kwakernaak and Sivan 1972). This allows the specification of boundaries for the design parameters which guarantee a nominally stable closed loop system. In the LQR approach, the controller gain vector $k$ is chosen in such a way that the control law $u=-k x$ minimises the cost function

$$
J_{c}(u)=\int_{t_{0}}^{t_{1}}\left[x^{T}(t) Q_{c} x(t)+u^{T}(t) R_{c} u(t)\right] d t
$$

subject to the system dynamics (1). Considering the steady state case $t_{1} \rightarrow \infty$, the optimisation problem (47) is solved using the corresponding algebraic Ricatti equation. A feedback 
gain vector $k$ can then be obtained by choosing the elements of the matrices $Q_{c}$ and $R_{c}$, and nominal stability can be guaranteed if these matrices are positive definite. As discussed in Section 5.1, the system model is second order, and we therefore choose to parametrise the design using two positive scalars, $q_{v}$ and $q_{p}$,

$$
R_{c}=1 \quad Q_{c}=\left[\begin{array}{cc}
q_{v} & 0 \\
0 & q_{p}
\end{array}\right], \text { with } q_{v}, q_{p}>0
$$

The observer gain vector $L$ is obtained by applying the same approach to the dual system $\left[A^{T}, C^{T}, B^{T}, D\right]$ (Kwakernaak and Sivan 1972). It was found that the results are relatively insensitive to observer properties which was therefore parametrised by a single positive variable, $q_{o}$,

$$
R_{o}=1 \quad Q_{o}=q_{o} B B^{T} \text { with } q_{o}>0
$$

where $R_{o}$ and $Q_{o}$ correspond to $R_{c}$ and $Q_{c}$ in equation (47) for the dual system.

The controller can then be fully specified by the positive parameter vector $\theta=\left[q_{v}, q_{p}, q_{o}, \Delta\right]$ (augmented by $\Delta_{o l}$ for intermittent control).

An optimisation criterion $J$ is defined is the weighted mean squared difference between the estimated FRF and its parametric fit

$$
J(\theta)=\frac{1}{N_{f}} \sum_{k=1}^{N_{f}} w_{k}^{2}\left[\hat{T}\left(j \omega_{k}\right)-\tilde{T}\left(j \omega_{k}, \theta\right)\right]^{2}
$$

The estimated coherence from equation (45) was chosen as the weighting factor, $w_{k}=$ $\hat{C}_{u d}\left(j \omega_{k}\right)$, as this penalises data depending on how much they are contaminated by noise. This criterion favours lower frequency data since $|T(j \omega)|$ tends to be larger in this range and, for experimental data, the coherence is smaller at higher frequencies. Other regularisation approaches are also possible.

Similar to the approach described in (Gawthrop et al. 2009), the parameter vector is separated into two parts, time delay parameters,

$$
\theta_{\Delta}= \begin{cases}{[\Delta]} & \text { for NPC and PC } \\ {\left[\Delta, \Delta_{o l}\right]} & \text { for IC }\end{cases}
$$

and controller design parameters

$$
\theta_{c}=\left[q_{v}, q_{p}, q_{o}\right]
$$

such that $\theta=\left[\theta_{\Delta}, \theta_{c}\right]$. The time delay parameters are varied over a predefined range, with the restriction that $\Delta_{o l}>\Delta$ (Gawthrop and Wang 2007). For each given set of time delay 
parameters, a corresponding set of optimal controller design parameters $\theta_{c}^{*}$ is found which solves the constrained optimisation problem

$$
\theta_{c}^{*}=\arg \min _{\theta_{c}} J\left(\left[\theta_{\Delta}, \theta_{c}\right]\right), \quad \theta_{c}>0
$$

In this work we chose the SQP algorithm as described by Nocedal and Wright (2006) and implemented in the Optimization Toolbox of MATLAB (Mathworks, USA) to solve (53) for each set of $\theta_{\Delta}$

The optimal cost function for each set of time-delay parameters, $J^{*}\left(\theta_{\Delta}\right)$, is calculated, and the overall optimum, $J^{*}$ is determined. For analysis, the time-delay parameters corresponding to the optimal cost are determined, with $\Delta$ and $\Delta_{o l}$ combined to give the effective time-delay for the IC (cf. Equation (14)),

$$
\Delta_{e}= \begin{cases}\Delta & \text { for NPC and PC } \\ \Delta+0.5 \Delta_{o l} & \text { for IC }\end{cases}
$$

\section{Simulation}

As a preliminary test, the identification procedures of Section 4 were applied to simulated data. In particular, the closed-loop system corresponding to a second-order unstable system was simulated with each of the three controllers and the resulting three sets of data were identified using parametric models for each of the three controllers in turn. Section 5.1 describes the system model used, Section 5.2 outlines how the simulation data was generated and Section 5.3 presents the results.

\subsection{System model}

The system to be controlled was chosen to be an unstable second order system approximating the dynamics of a standing human (Loram et al. 2009). The instability meant that the system had to be actively controlled to be stabilised.

The system was of the form of Equations (1)-(2) with

$$
\begin{aligned}
& \dot{x}(t)=\left[\begin{array}{cc}
-0.0372 & 1.231 \\
1 & 0
\end{array}\right] x(t)+\left[\begin{array}{c}
6.977 \\
0
\end{array}\right][u(t)-d(t)] \\
& y(t)=\left[\begin{array}{ll}
0 & 1
\end{array}\right] x(t)
\end{aligned}
$$


The physical meaning of the system input $u(t)$ is muscle shortening applied at the end of the compliant tendon, while the output $y(t)$ (the second state) is the position of the pendulum. The first state represents the angular velocity of the pendulum. Full details of the setup the derivation of the system model are given in Loram et al. (2009).

Taking the physical meaning of the two system states into account, the elements of the controller design matrix $Q_{c}$ (equation (48)) can be interpreted as weighting factors for control of velocity $\left(q_{v}\right)$ and position $\left(q_{p}\right)$, respectively.

\subsection{Simulation method}

Simulation data was generated for the three controller cases described in section 2: nonpredictive control (NPC), predictive control (PC) and intermittent control (IC). The design parameters were

$$
\Delta=100 \mathrm{~ms}, \quad q_{v}=1, \quad q_{p}=1, \quad q_{o}=100
$$

for all three designs, with $\Delta_{o l}=250 \mathrm{~ms}$ for the intermittent controller.

Periodic identification data were generated for an unpredictable multisine disturbance input $d\left(t_{n}\right)$ (equation (41) with $N_{f}=100, f_{0}=0.1 \mathrm{~Hz}$, implying a period duration of $T_{p}=$ $10 \mathrm{sec}$ ). Simulations of $100 \mathrm{sec}$ duration (i.e. 10 periods) with a sample period of $T_{s}=0.01 \mathrm{sec}$ were performed. Using the equations derived in section 3 , the closed loop frequency responses $T\left(j \omega_{k}\right)$ were calculated. Due to the periodic nature of the disturbance signal, the resulting control signal could be obtained as

$$
u\left(t_{n}\right)=\sum_{k=1}^{N_{f}}\left|T\left(j \omega_{k}\right)\right| \cos \left(\omega_{k} t_{n}+\angle T\left(j \omega_{k}\right)+\phi_{k}\right)
$$

An extract over three periods $(30 \mathrm{sec})$ of the simulation data generated in this way for the predictive controller is shown in figure 2.

\subsection{Simulation results}

In a first step, we apply the system identification procedure to the three simulation data sets, where the parameters used to generate them are known (equation (57)). The simulations are defined by their controller structure: non-predictive control (NPC sim), predictive control (PC sim) and intermittent control (IC sim). 

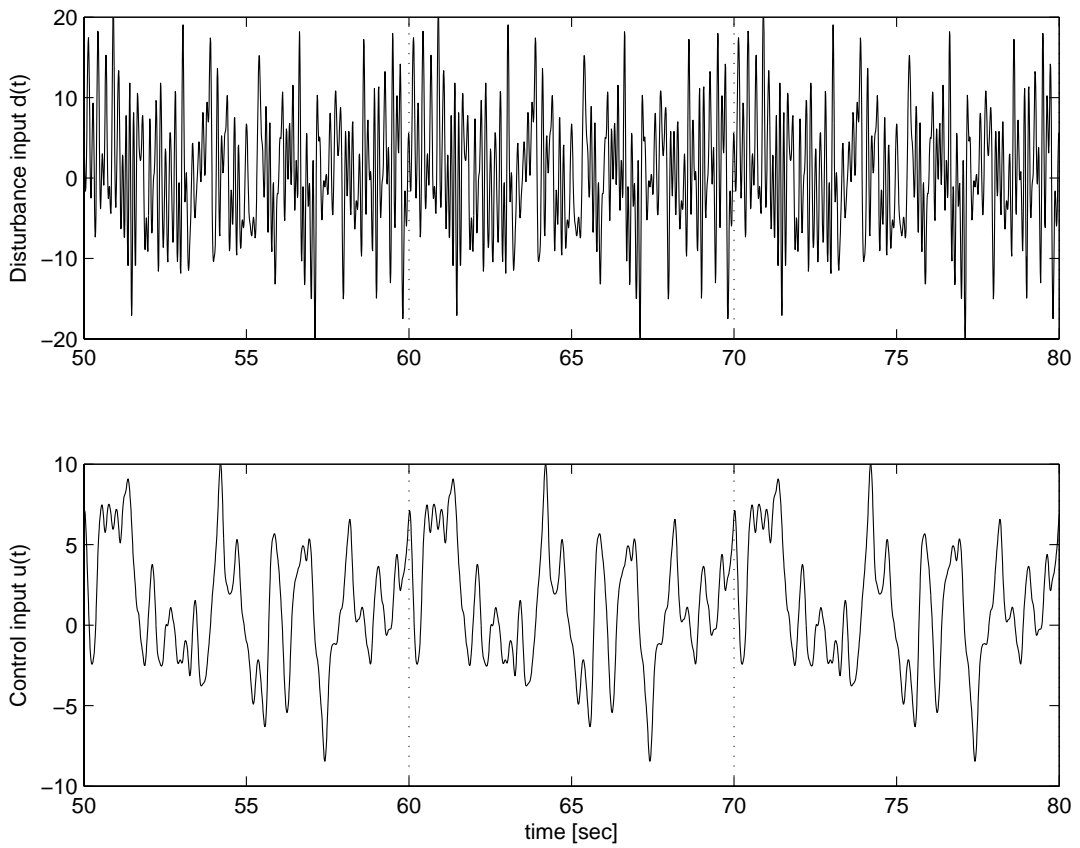

Fig. 2 Simulation data generated for the predictive controller. The vertical dashed lines indicate the periods of $10 \sec$ duration.

As outlined in section 4.2 , the time delay $\Delta$ is varied (range $[0, \ldots, 400] \mathrm{ms}$ in increments of $10 \mathrm{~ms}$ ) and the corresponding optimal set of controller design parameters, $\theta_{c}^{*}$ (equation (53)), is determined together with the value of the cost function $J^{*}\left(\theta_{\Delta}\right)$. For the intermittent controller structure, in addition the intermittent interval, $\Delta_{o l}$ is varied over the same range as $\Delta$, and for each value of $\Delta$, the intermittent interval which results in the lowest value of the cost function $J$ is chosen and denoted as $\Delta_{o l}^{*}$.

The results in terms of the minimal value of the optimisation criterion $J^{*}\left(\theta_{\Delta}\right)$ as a function of the loop time delay $\Delta$ are summarised in figure 3 . The bold marks in each sub-figure indicate the minimal cost function value achieved for this controller and the corresponding time delay $\Delta^{*}$. It can be seen that for each of the three simulations, the corresponding controller used for the design was identified correctly, i.e. a perfect fit was achieved at $\Delta=100 \mathrm{~ms}$. As expected the two other controller structures were unable to perfectly match the data. The goodness of fit for the predictive controller and the intermittent controller are similar while the non-predictive controller is unable to satisfactorily approximate the data generated with the other two controller architectures. 


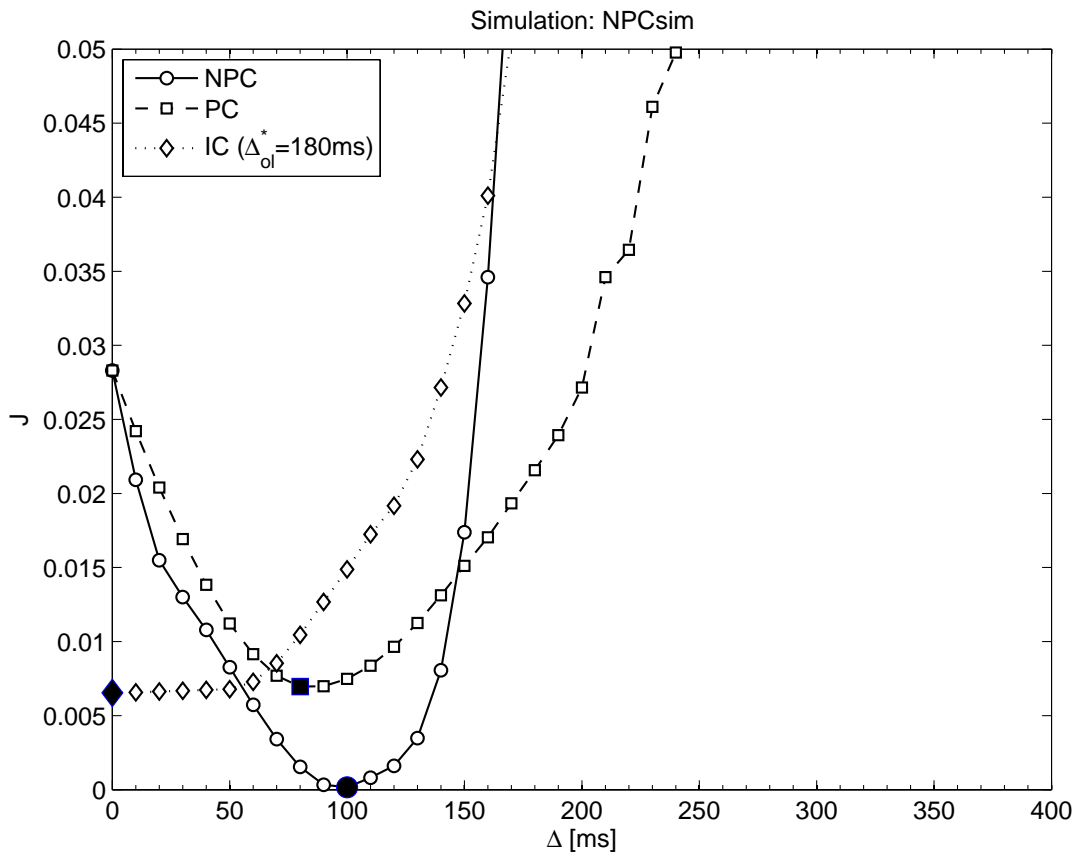

(a) Simulation with non-predictive controller. The effective optimal delay for IC is $\Delta_{e}^{I C}=90 \mathrm{~ms}$.

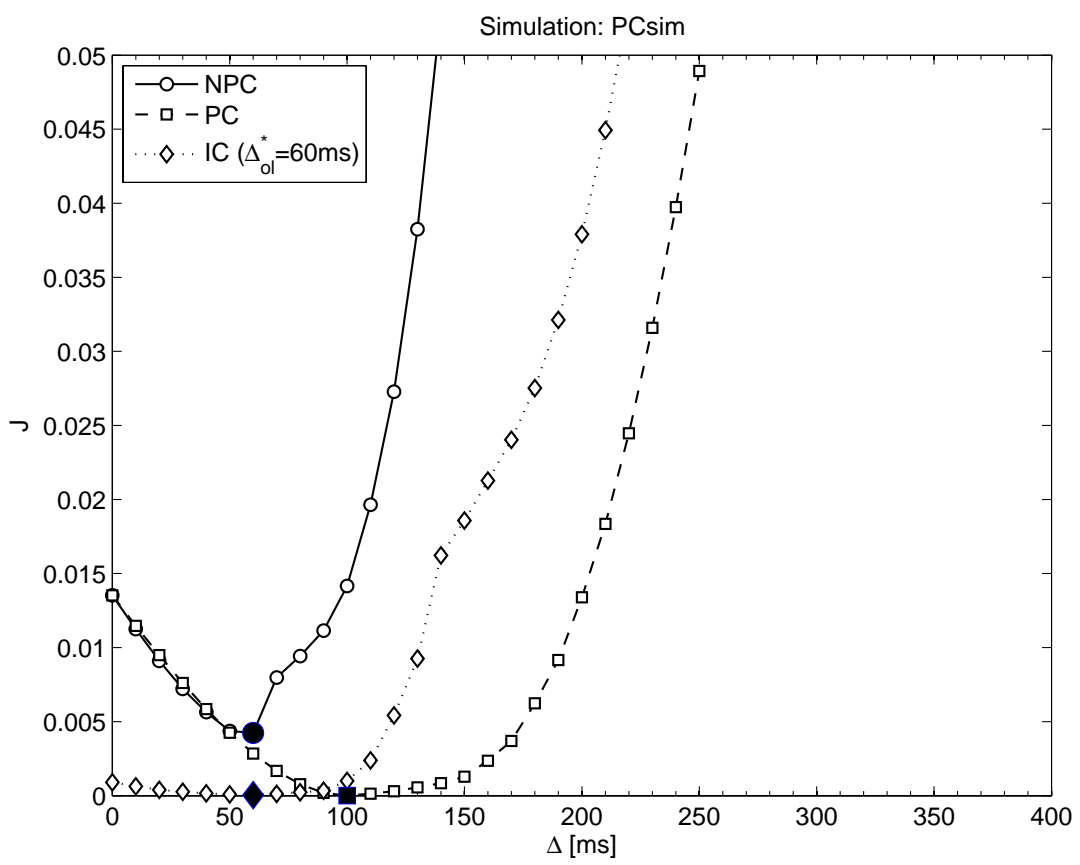

(b) Simulation with predictive controller. The effective optimal delay for IC is $\Delta_{e}^{I C}=120 \mathrm{~ms}$.

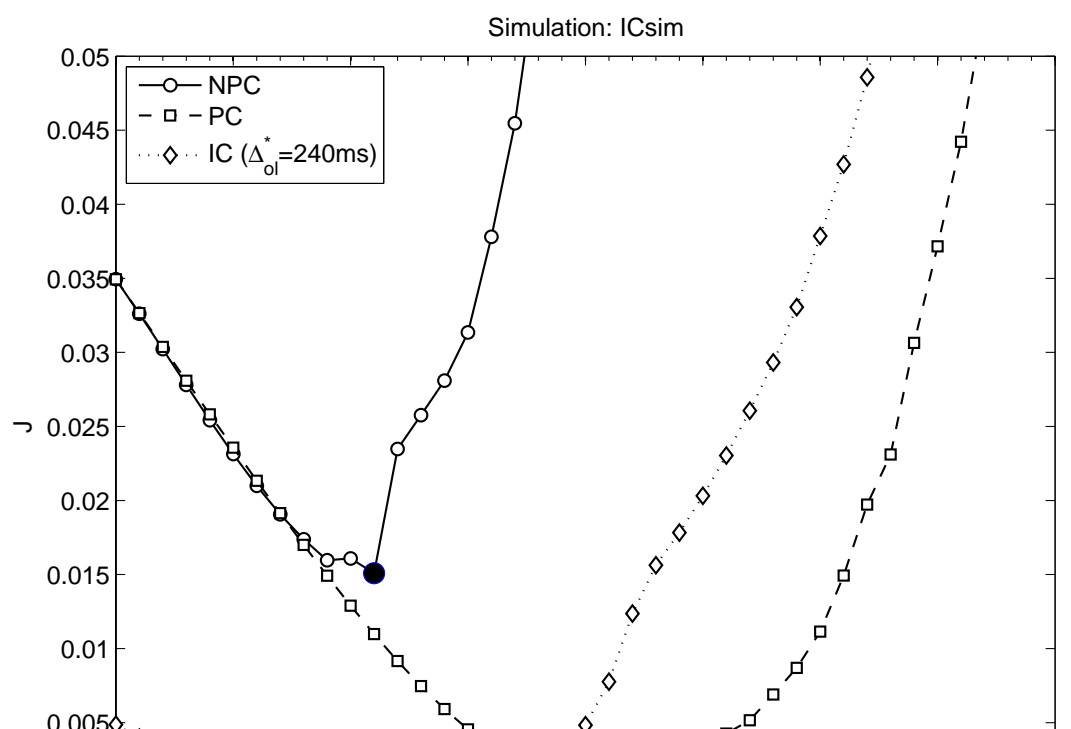


The value of the optimal time delay $\Delta^{*}$ obtained for the predictive controller is generally larger than that identified for the intermittent controller. When the effective time-delays (Equation (54)) are considered then the values corresponding to the optima are very similar for the predictive controller and the intermittent controller, for all three simulations. The time delay identified for the non-predictive controller is smaller than (or equal to) those for the other two controllers in the cases of the PC simulation and the IC simulation. For the non-predictive controller it can also be observed that the cost value increases sharply as $\Delta$ increases. This is due to the fact that the closed loop tends to be destabilised as the time-delay increases, since, unlike for the intermittent and predictive controllers, $\Delta$ is not explicitly taken into account when designing the controller.

To illustrate the procedure behind the optimisation performed, figure 4 shows the plots of the estimated frequency response functions, $\hat{T}\left(j \omega_{k}\right)$, together with the optimised frequency responses, $\tilde{T}\left(j \omega_{k}, \theta^{*}\right)$, for each controller in the complex plane, for all three simulated data sets. Figure 5 shows the impulse responses estimated from these frequency responses. These results confirm that the FRFs and impulse responses of the predictive and the intermittent controllers are almost indistinguishable, while the non-predictive controller results are clearly different.

\section{Experiments}

Experiments were conducted in which volunteers where instructed to control a dot on the screen (the system output) using a joystick (representing the system input). The same simulated unstable system as described in Section 5.1 was used. The similarity of the system model to the human balance problem meant that it was possible for the human to control the system without prior practice. The resulting data is analysed in exactly the same way as the simulated-controller data of Section 5. Section 6.1 describes the experimental method in more detail, and Section 6.2 shows the experimental results.

\subsection{Experimental method}

The experimental procedures and methods were based on those described in Loram et al. (2009). Eleven healthy adults (9 male, 2 female, aged between 21 and 59 years ( $36 \pm 13$ years mean \pm s.d.)) were using a sensitive, contactless, uniaxial joystick to control the left right 


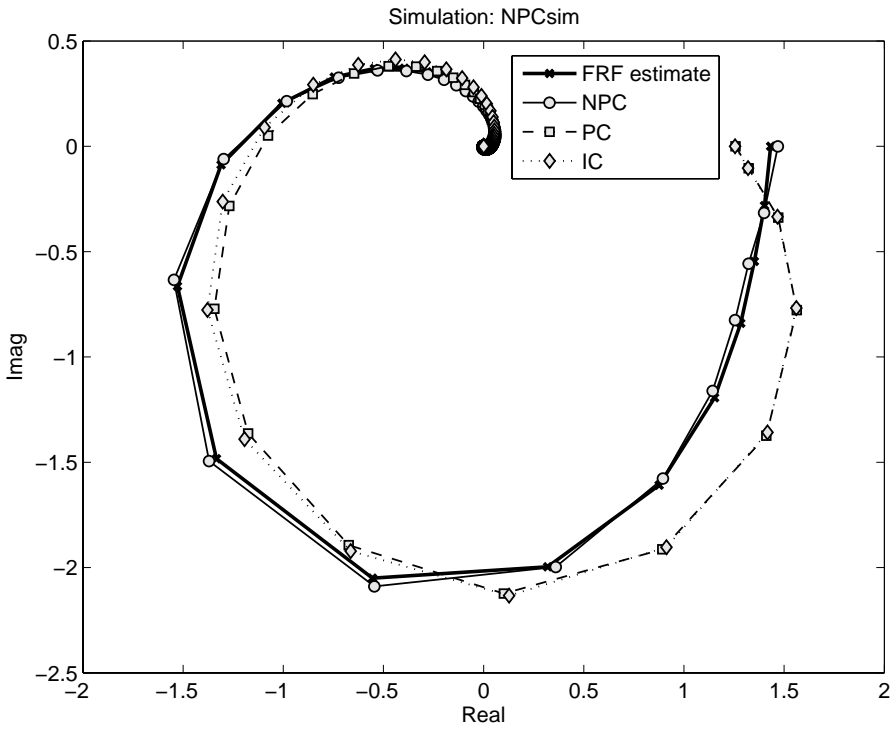

(a) Simulation with non-predictive controller.

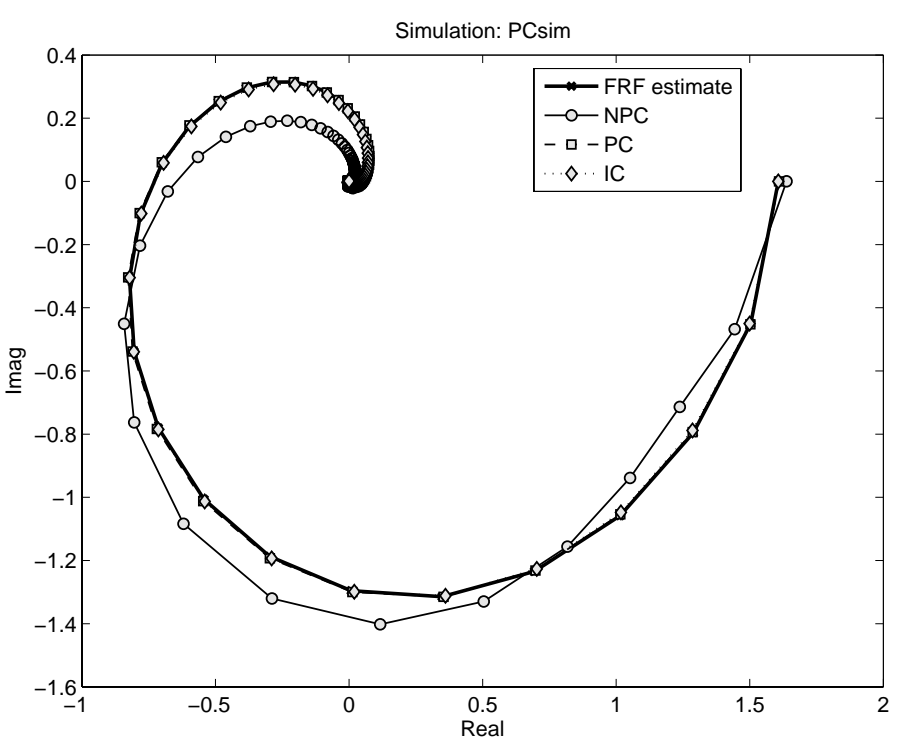

(b) Simulation with predictive controller.

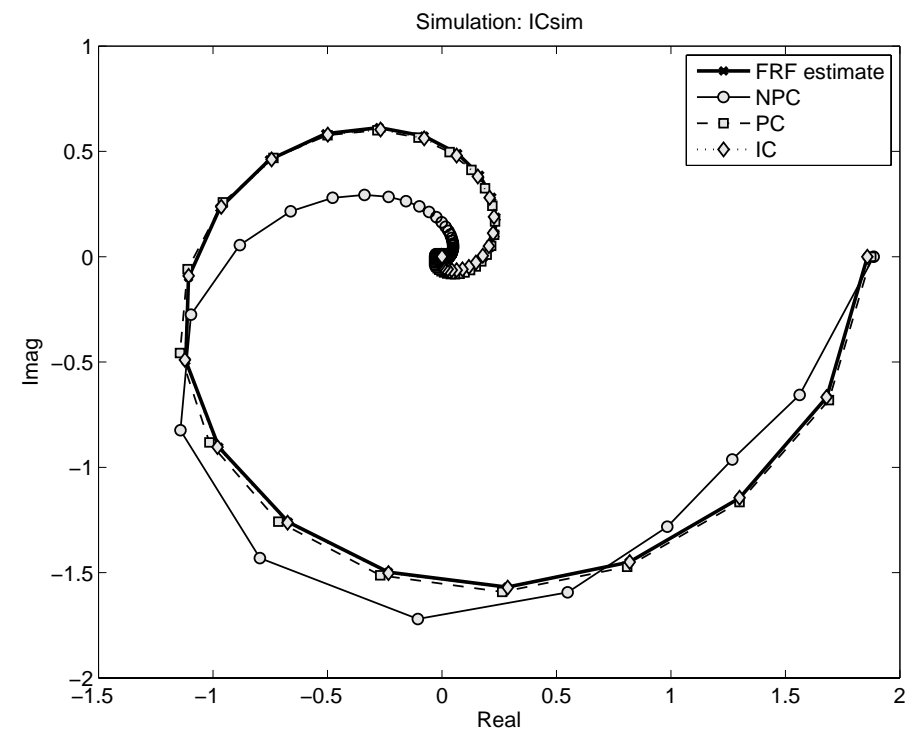

(c) Simulation with intermittent controller.

Fig. 4 Closed-loop nyquist plots of estimated frequency responses, $\hat{T}$ (bold solid line) and the fitted responses $\tilde{T}$ for the different controller structures (NPC: solid, PC: dashed and IC: dotted lines). The markers indicate the values at the discrete frequencies $\omega_{k}$. 


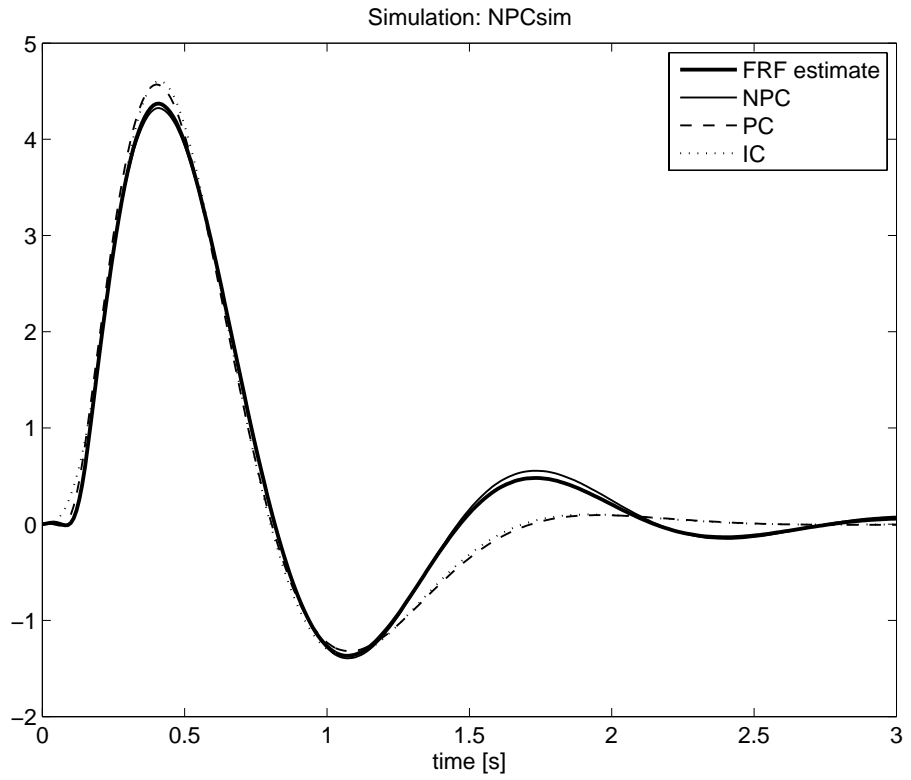

(a) Simulation with non-predictive controller.

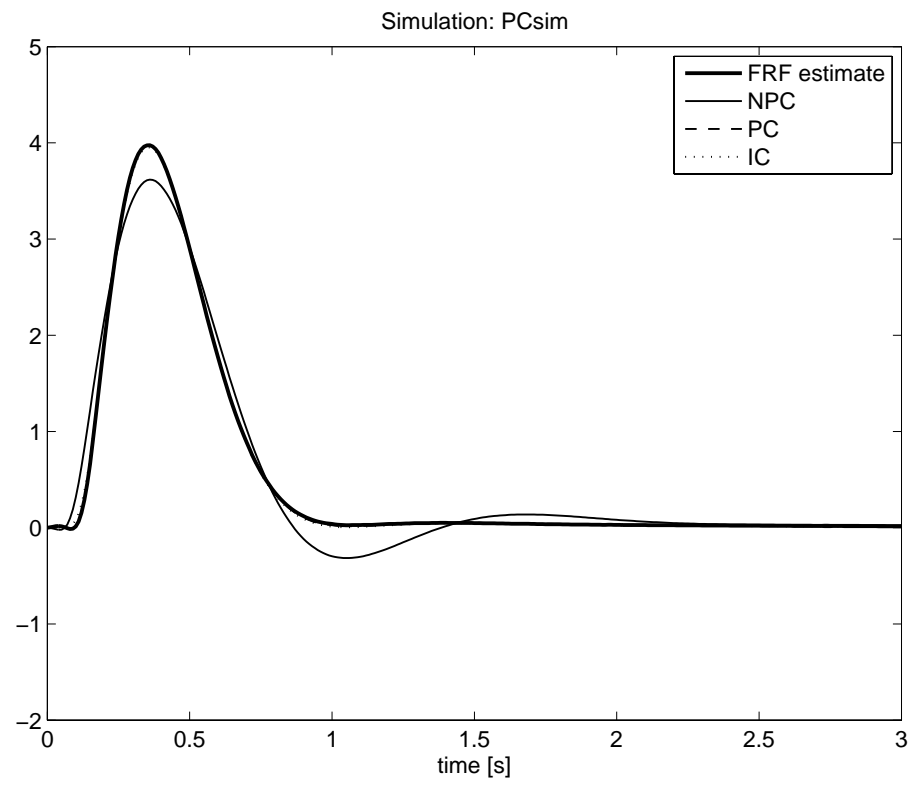

(b) Simulation with predictive controller.

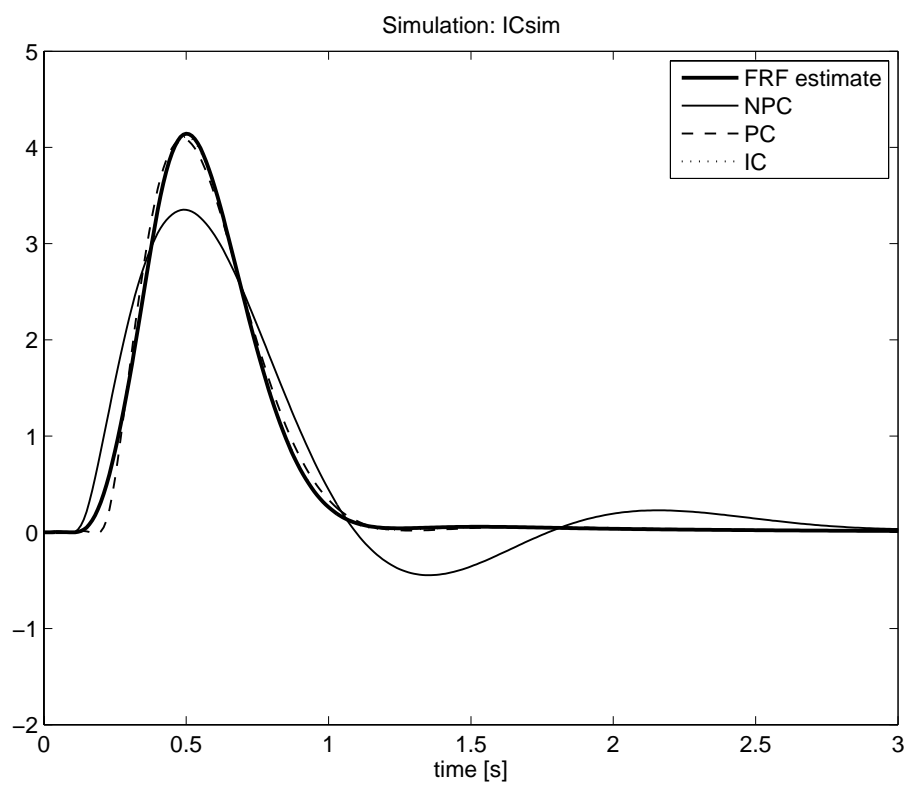

(c) Simulation with intermittent controller. 
position of a dot on an oscilloscope screen. The joystick position corresponded to the input to the 2nd order unstable model of Section 5.1 while the dot indicated the position of the simulated pendulum. The virtual pendulum load was subject to an unpredictable multisine disturbance $d(t)$, containing frequencies between 0.1 and $10 \mathrm{~Hz}\left(N_{f}=100\right.$ and $f_{0}=0.1 \mathrm{~Hz}$ in equation (41)). Data was recorded at a sample period of $T_{S}=0.01 \mathrm{sec}$, and the duration of one trial was $200 \mathrm{sec}$. For the identification procedure, the first $20 \mathrm{sec}$ of data were discarded, resulting in 18 periods ( $180 \mathrm{sec})$ available for analysis.

While the primary objective of the participants was to stabilise the load throughout the trial, they were also instructed to apply one of two control strategies:

i) Position control: keep the load position as close as possible to the centre of the oscilloscope screen.

ii) Velocity control: minimise the velocity of the load, while the position on the screen is secondary.

Examples of experimental data for 3 periods (30 seconds) are shown in figure 6 for both control strategies.

\subsection{Experimental results}

The same identification procedure as descibed in Section 5.3 was applied. Figures 7 and 8 show detailed results for one subject for data obtained during position control and velocity control trials, respectively. Figure 7 (a) and $8\left(\right.$ a) depict the best fit values, $J^{*}\left(\theta_{\Delta}\right)$, for each time delay $\Delta$. Predictive and intermittent control result in similar minimal fit values and effective time delays (eq. (54)), while the non-predictive control structure has a smaller time delay with a slightly larger minimal fit.

The complex-plane frequency response plots are shown in figures 7(b) and 8(b). The frequency responses for predictive and intermittent controllers are almost indistinguishable, while the response with the non-predictive controller is different, particularly at higher frequencies (i.e. close to the origin). The impulse responses derived from the frequency response functions (shown in figures 7(c) and 8(c)) confirm that the approximations obtained with the intermittent and predictive controllers are similar to each other and close to the FRF estimate, while that derived with the non-predictive controller results in a markedly different approximation. 

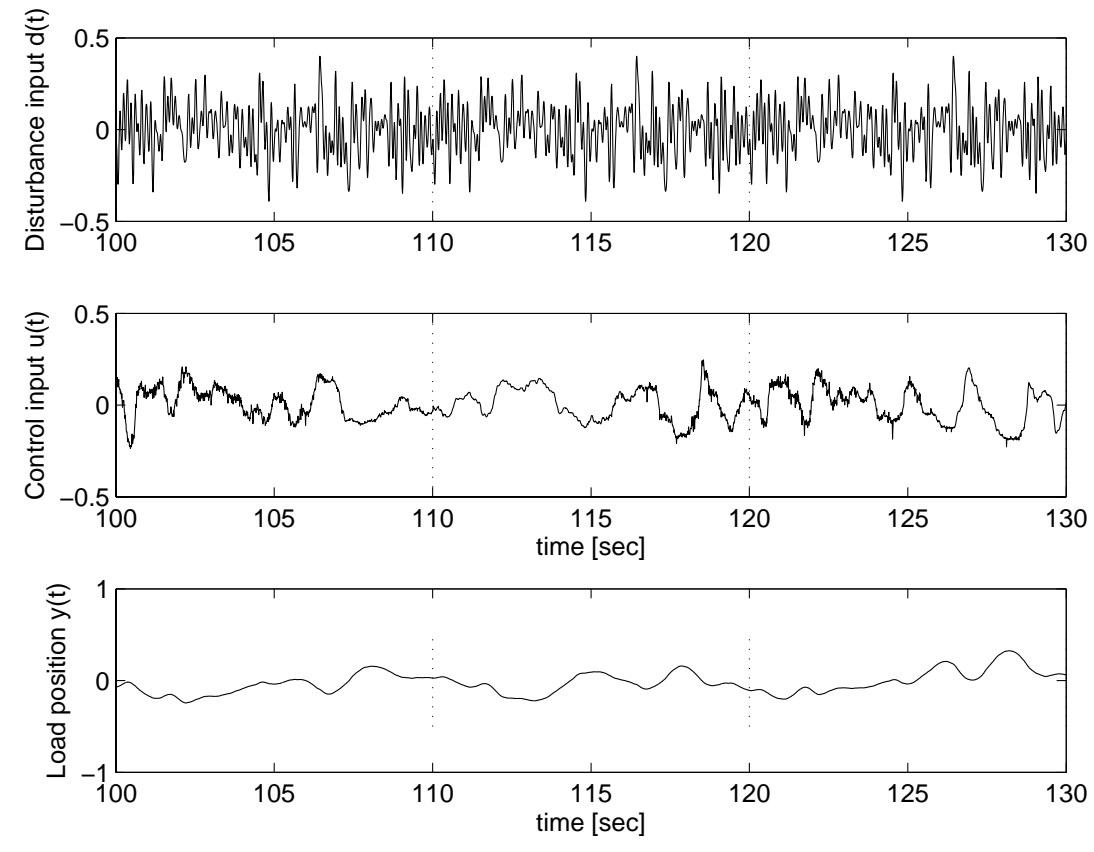

(a) Experiment, Subject 7, position control
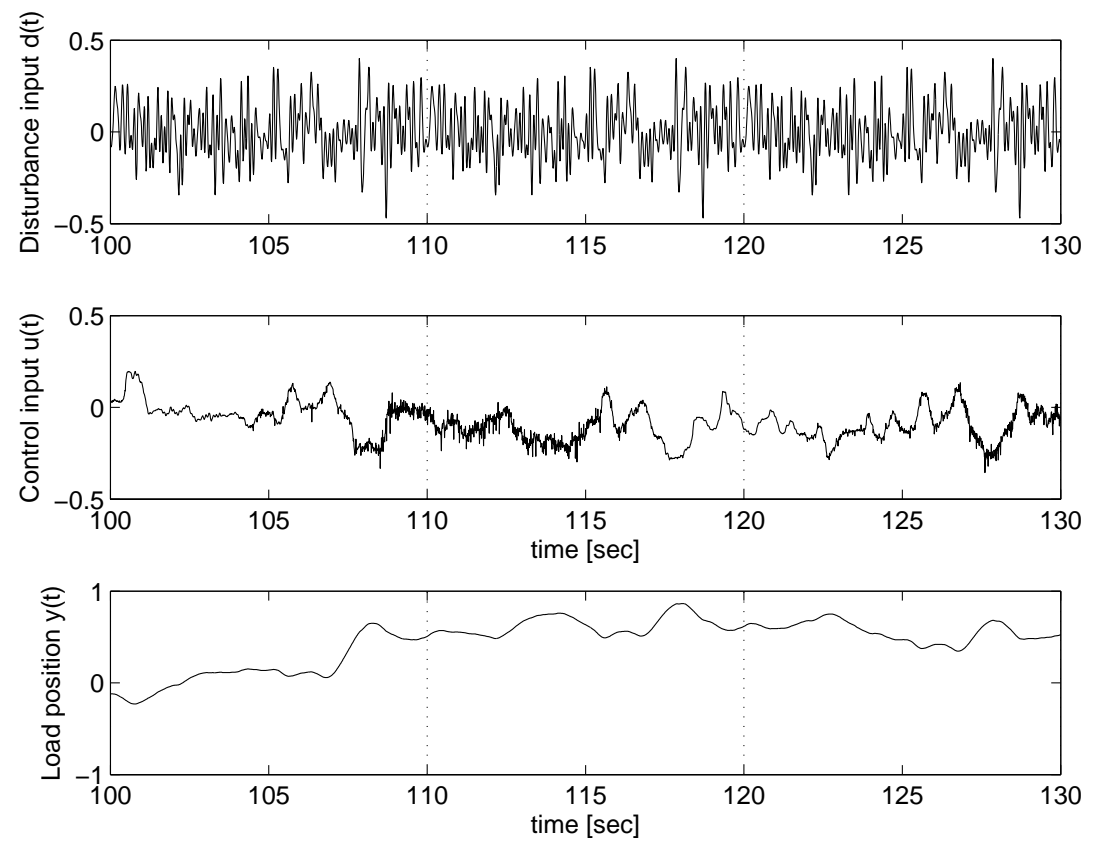

(b) Experiment, Subject 7, velocity control

Fig. 6 Experimental data. Position control keeps the load position close to zero, while velocity control attempts to minimise variations in load position. The vertical dashed lines indicate the periods of $10 \mathrm{sec}$ duration. 


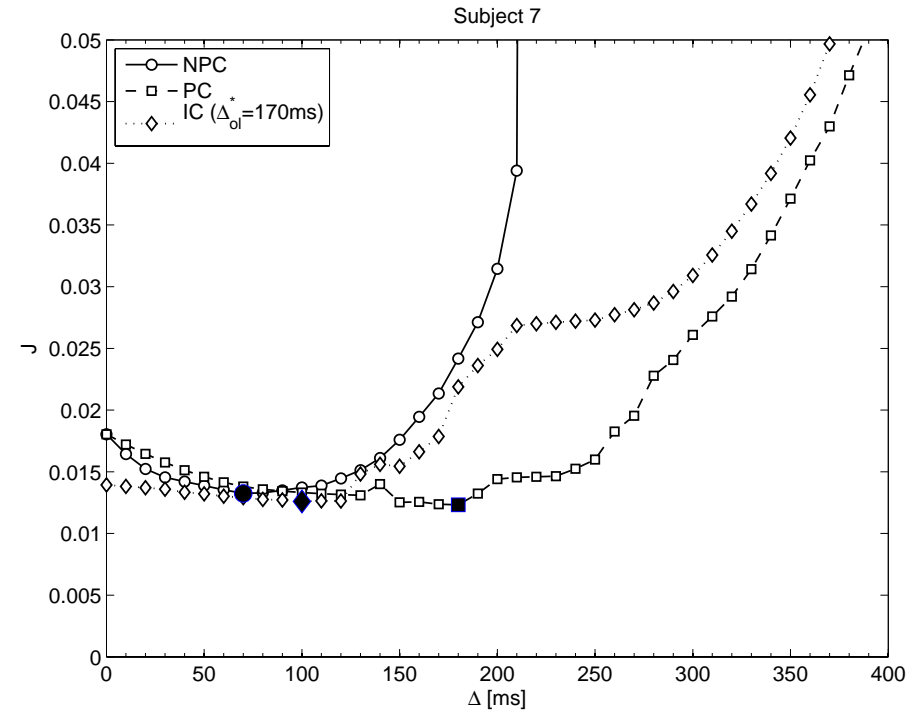

(a) Optimisation criterion $J$ as a function of the time delay $\Delta$, for different identified controller structures. The bold markers indicate the minima of the optimisation criterion for the corresponding controller structure. For the intermittent controller the intermittent interval $\Delta_{o l}^{*}$ corresponding to the minimum of $J$ is given in the legend and the effective optimal delay is $\Delta_{e}^{I C}=185 \mathrm{~ms}$.

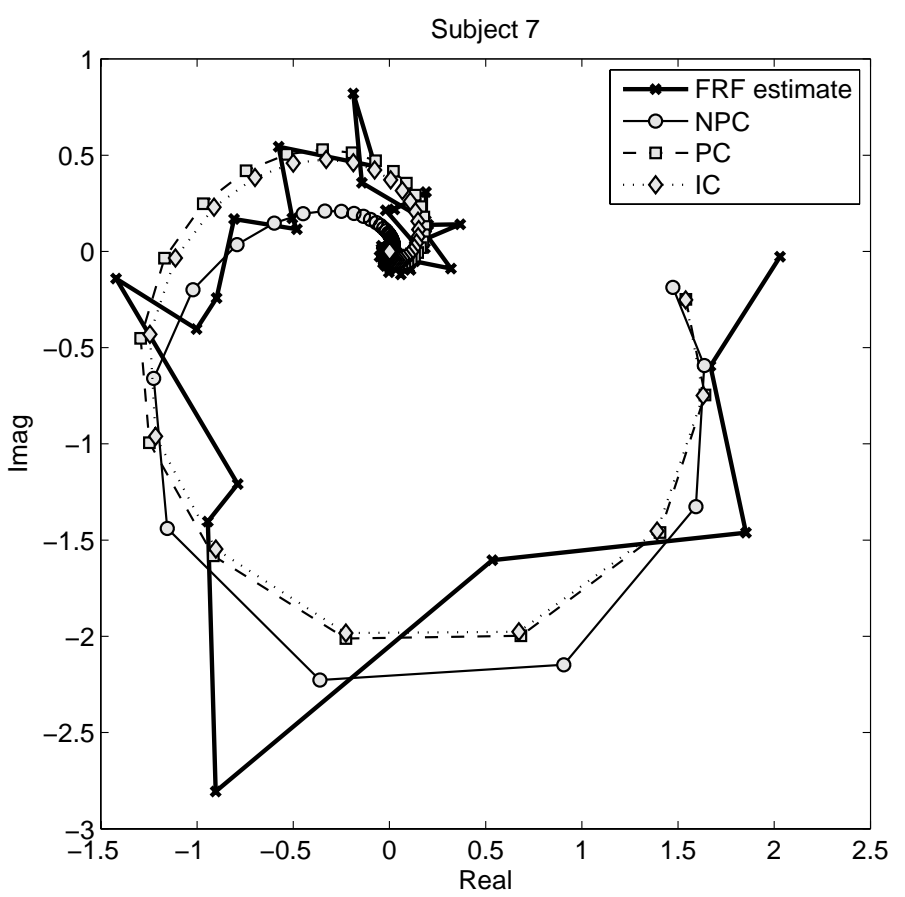

(b) Closed-loop nyquist plots of the estimated frequency response function $(\hat{T}$, bold solid line) and the fitted responses $(\tilde{T})$ for the different controller structures.

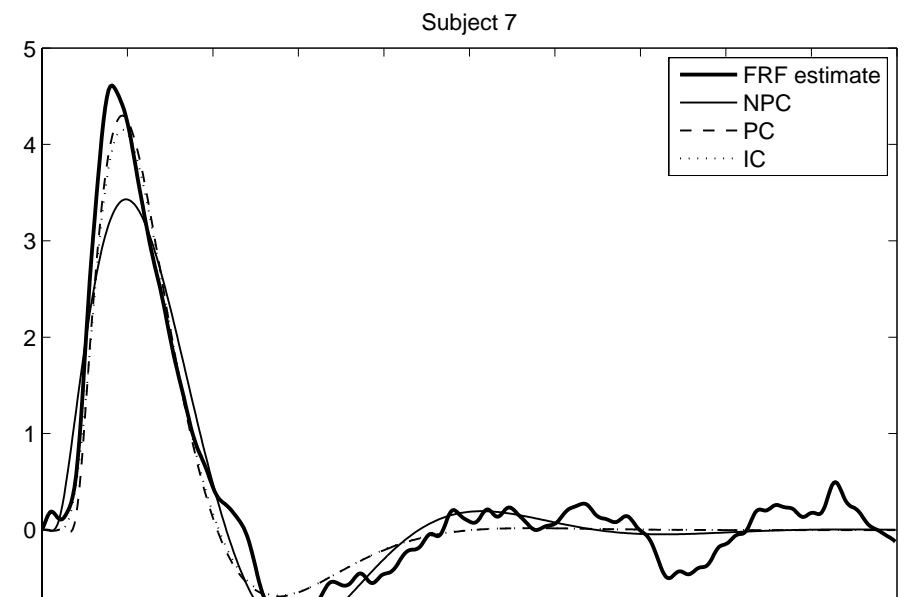




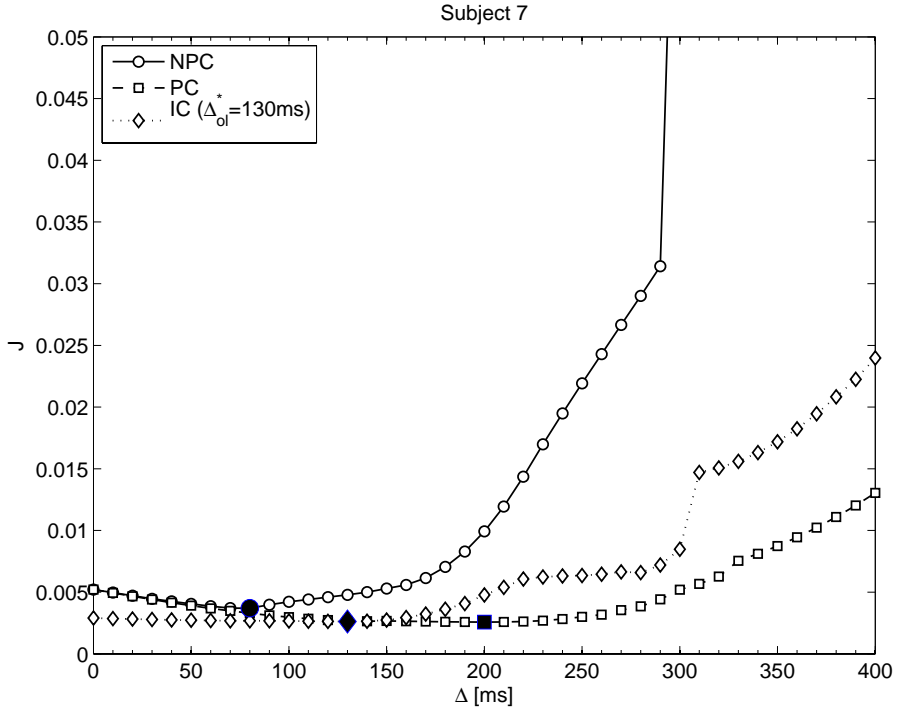

(a) Optimisation criterion $J$ as a function of the time delay $\Delta$, for different identified controller structures. The bold markers indicate the minima of the optimisation criterion for the corresponding controller structure. For the intermittent controller the intermittent interval $\Delta_{o l}^{*}$ corresponding to the minimum of $J$ is given in the legend and the effective optimal delay is $\Delta_{e}^{I C}=195 \mathrm{~ms}$.

Subject 7

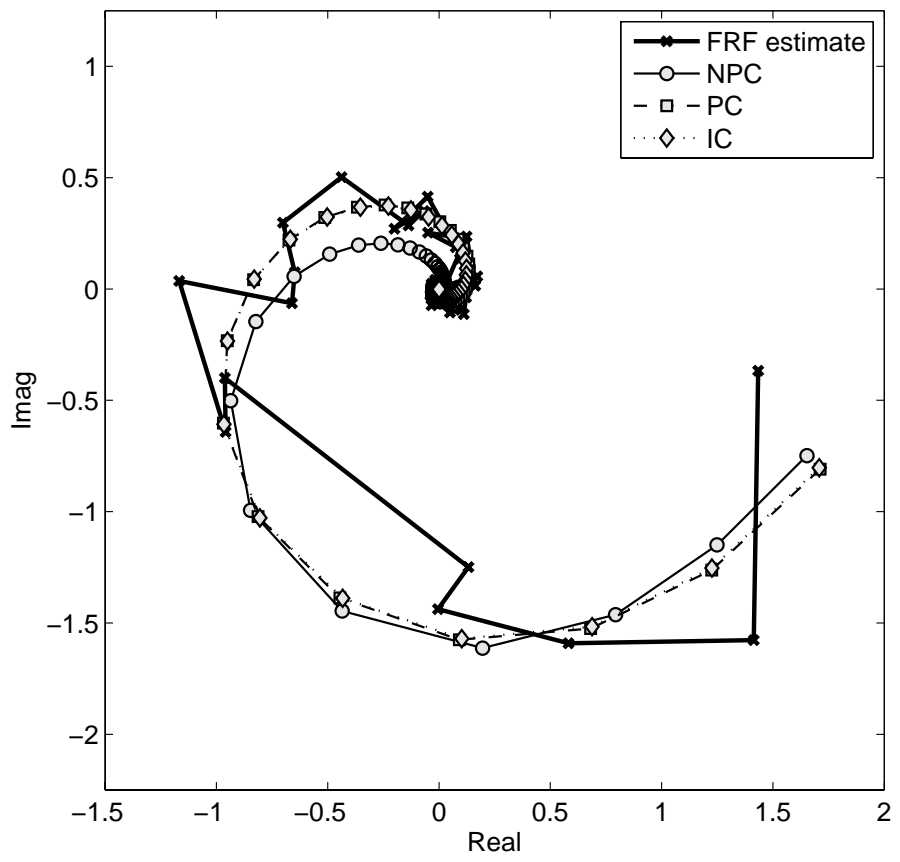

(b) Closed-loop nyquist plots of the estimated frequency responses $(\hat{T}$, bold solid line) and the fitted responses $(\tilde{T})$ for the different controller structures.

Subject 7

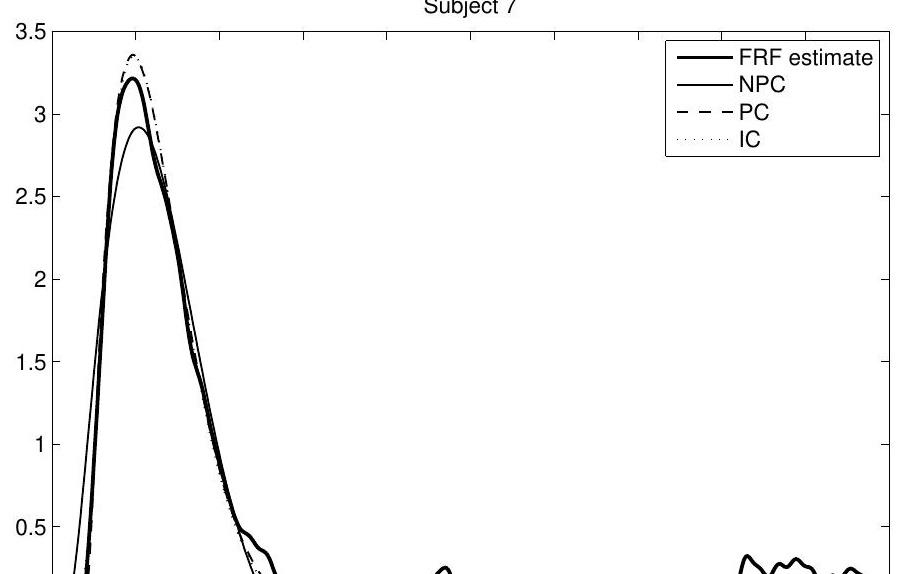


Results for all subjects are shown in figures 9 and 10. Figure 9 summarises the best fit values, $J\left(\theta^{*}\right)$, obtained for the three controller structures. It shows that for both control strategies (position and velocity control), the intermittent and the predictive controllers result in a closed loop which can fit the measured data in a similar way. The fit values obtained with the non-predictive controller are slightly worse for almost all subjects. The mean values of the best fit for each controller (shown as straight horizontal lines) confirm this.

The effective time delays which correspond to the best fit values are summarised in figure 10. The optimal time delays obtained for the predictive controller and the intermittent controllers are very similar and consistently larger than those for the non-predictive controllers, independent of the adopted control strategy. This is also evident in the mean time delays over all subjects, shown as straight horizontal lines. Comparing the control strategies, it appears that velocity control (figure 10(b)) results in slightly smaller time delays than position control (figure 10(a)).

Figure 10 also shows the values for the optimal intermittent interval for each subject.

Table 1 summarises the optimal parameter values obtained for all subject for the three control structures. Although the standard deviations are very large which reflects the significant inter-subject variability, the results show a number of trends, independent of the controller structure: (i) the controller design parameter $q_{p}$ is larger for position control than for velocity control; (ii) the controller design parameter $q_{v}$ is smaller for position control than for velocity control; (iii) the effective time delay $\Delta_{e}$ is slightly smaller for velocity control than for position control (this was already discussed above). (iv) for the intermittent controller, the intermittent interval $\Delta_{o l}$ is slightly smaller for velocity control than for position control. As mentioned earlier, the controller parameter $q_{p}$ is the second element in the matrix $Q_{c}$ (cf. equation (48)) and therefore represents the weight associated with the second state (position). Similarly, $q_{v}$ is associated with the first state (velocity). The optimised parameters therefore appear to reflect the control strategy adopted by the subject: for position control a larger weight is put on the 2 nd state (position) while for velocity control a larger weight is associated with the first state (velocity). The value of the observer parameter $q_{o}$ appears to be unrelated to the control strategy. 


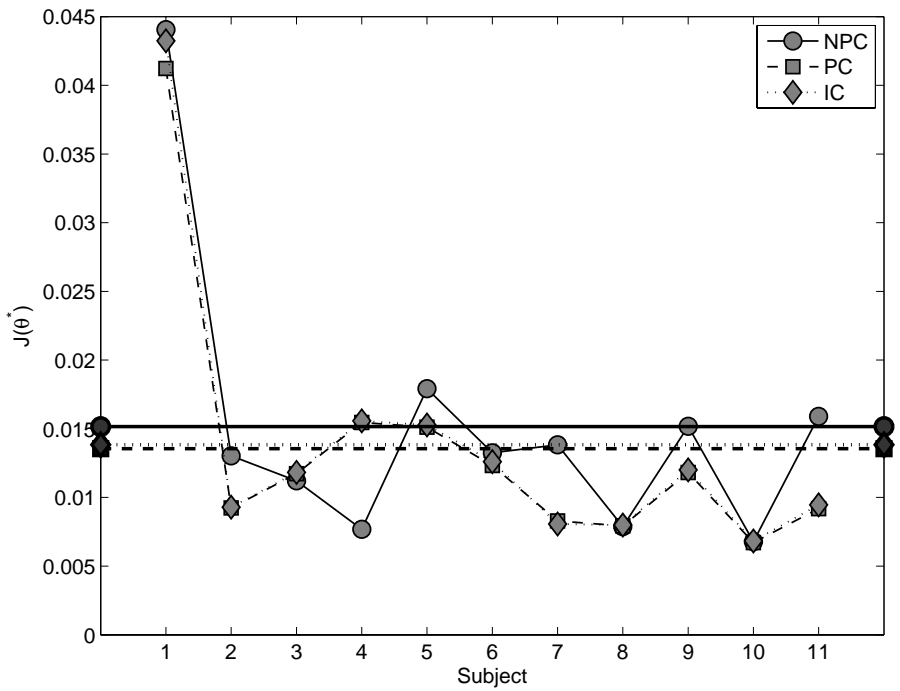

(a) Position control

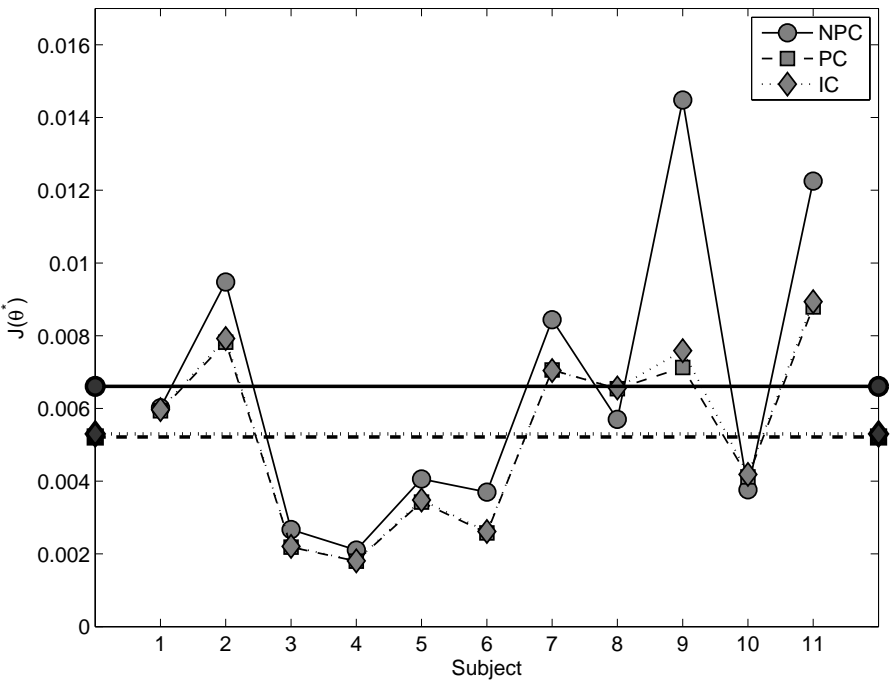

(b) Velocity control

Fig. 9 Best fit values, $J\left(\theta^{*}\right)$, for both control strategies, for each subject. The horizontal lines indicate the mean value over all subjects for each controller structure.

\section{Conclusions}

A well-established two stage frequency domain process has been used to identify controllers from closed loop simulation and experimental data. While this approach is an established 


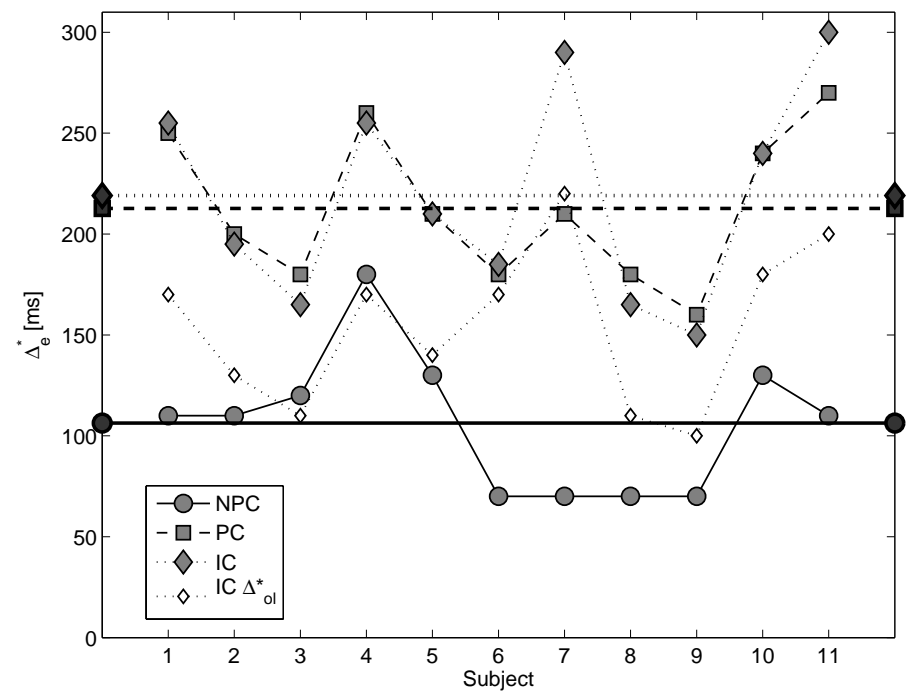

(a) Position control

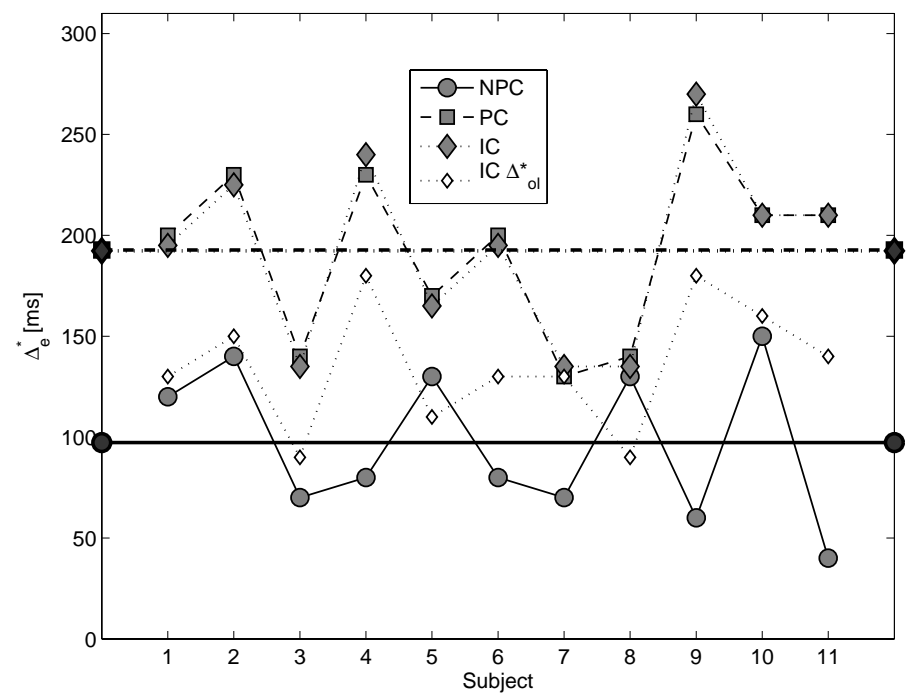

(b) Velocity control

Fig. 10 Time delay, $\Delta^{*}$, corresponding to the best fit values shown in figure 9 , for both control strategies, for each subject. The horizontal lines indicate the mean value over all subjects for each controller structure. In addition, the optimal intermittent interval, $\Delta_{o l}^{*}$, is shown for each subject. 


\begin{tabular}{|c|c|c|c|c|}
\hline & & NPC & $\mathrm{PC}$ & IC \\
\hline \multirow{2}{*}{$q_{p}$} & pos & $9.0 \pm 15.2$ & $42.4 \pm 63.4$ & $51.1 \pm 82.3$ \\
\hline & vel & $2.1 \pm 4.3$ & $13.1 \pm 12.4$ & $15.9 \pm 16.1$ \\
\hline \multirow{2}{*}{$q_{v}$} & pos & $0.6 \pm 1.1$ & $0.4 \pm 1.0$ & $0.8 \pm 2.1$ \\
\hline & vel & $0.8 \pm 0.8$ & $5.3 \pm 12.0$ & $6.7 \pm 14.8$ \\
\hline \multirow{2}{*}{$q_{o}$} & pos & $136.2 \pm 206.2$ & $92.4 \pm 168.3$ & $470.9 \pm 983.9$ \\
\hline & vel & $31.5 \pm 22.5$ & $275.4 \pm 614.6$ & $409.4 \pm 984.7$ \\
\hline \multirow{2}{*}{$\Delta_{e}[\mathrm{~ms}]$} & pos & $106 \pm 35$ & $213 \pm 37$ & $219 \pm 52$ \\
\hline & vel & $97 \pm 37$ & $193 \pm 42$ & $192 \pm 45$ \\
\hline \multirow{2}{*}{$\Delta_{o l}[\mathrm{~ms}]$} & pos & & & $155 \pm 39$ \\
\hline & vel & & & $135 \pm 31$ \\
\hline
\end{tabular}

Table 1 Optimised parameters (mean \pm standard deviation).

technique for linear time-invariant control structures such as non-predictive and predictive control, for which FRFs can be readily derived, it has been used here for the first time for intermittent control, based on the results of (Gawthrop 2009). The method is found to be robust even when used with physiological data which are noisy, non-linear and have high inter-subject variability. This is illustrated by the consistency of the best fit results for all 11 subjects (cf. figure 9), and by the concave shape of the cost functions for the experimental data (cf. figure 7 and 8) which have a clear minimum and are similar to those for the simulated data (cf. figure 3). It was noted that optimisation using the non-predictive control model is more problematic due to the unstable closed-loop associated with some parameter values; this problem does not occur in the other two cases provided that all of the parameters were constrained to be positive.

Three controller structures were compared: non-predictive control, predictive control and intermittent predictive control. The method was verified using simulated data and, in this case, could distinguish the three approaches although the difference between predictive and non-predictive was greater than that between intermittent and continuous predictive control.

The experimental results show that the best fit values are consistently worse for nonpredictive control than for the two predictive control structures; this verifies earlier results (Gawthrop et al. 2009) comparing non-predictive and predictive continuous control, though the results are more clear cut using the approach of this paper. 
Two experimental conditions were considered, position control and velocity control. The controller parameters identified reflect these conditions: for position control, the weighting factor $q_{p}$ associated with the position state is increased, while for velocity control, the weighting factor $q_{v}$ associated with the velocity state is larger. This could be observed consistently for all three controller structures.

For both the simulated data and the experimental data, the non-predictive controller structure results in effective time delays values which are significantly smaller than those obtained with predictive control and intermittent control. Only the predictive and intermittent controllers resulted in time delays which are in agreement with previous estimates (Loram et al. 2009; Gawthrop et al. 2009; Loram et al. 2011), and confirms the robustness of this method when used with experimental data.

From the results it appears that predictive and intermittent predictive controllers are equally valid descriptions of the human balancing control task investigated here. Both approaches allow fitting the estimated non-parametric frequency responses with comparable quality. This implies that the experimental data can be equally well explained using continuous predictive and intermittent predictive control hypotheses. This result is particularly interesting as it means that experimental results showing good fit for continuous predictive control models, dating back to at least those of Kleinman et al. (1970), do not rule out an intermittent explanation. A theoretical explanation for this result is given in (Gawthrop et al. 2011, Section 4.3) where the masquerading property of intermittent control is discussed: As shown there, the frequency response of an intermittent controller and that of the corresponding predictive controller are indistinguishable at lower frequency and only diverge at higher frequencies where aliasing occurs. As aliasing is due to the open-loop interval, its effect would be more obvious in the case when the plant dynamics and controller bandwidth were small compared to the intermittent interval. For our experimental conditions, however, where the pendulum dynamics are similar to those of human standing, the responses of the predictive and the intermittent controllers would be difficult to distinguish, and therefore both explanations appear to be equally valid.

\section{Acknowledgements}

The work reported here was supported by the linked EPSRC Grants EP/F068514/1, EP/F069022/1

and EP/F06974X/1 “Intermittent control of man and machine”. Peter Gawthrop was a Lever- 
hulme Emeritus Research Fellow when this work was initiated, and he gratefully acknowledges the support of the Leverhulme Trust. The authors wish to thank Dr Martin Lakie, School of Sport and Exercise Sciences, University of Birmingham, for his contributions to the experimental aspects of this work.

\section{References}

A. V. Alexandrov, A. A. Frolov, F. B. Horak, P. Carlson-Kuhta, and S. Park. Feedback equilibrium control during human standing. Biol. Cybern., 93:309-322, November 2005.

S. Baron, D. L. Kleinman, and W. H. Levison. An optimal control model of human response. Part II: Prediction of human performance in a complex task. Automatica, 6:371-383, May 1970.

R. T. Bye and P. D. Neilson. The BUMP model of response planning: Variable horizon predictive control accounts for the speed-accuracy tradeoffs and velocity profiles of aimed movement. Hum. Movement Sci., 27(5):771 - 798, 2008.

K. J. Craik. Theory of human operators in control systems. I. The operator as an engineering system. Brit. J. Psychol., 38:56-61, 1947a.

K. J. Craik. Theory of human operators in control systems. II. Man as an element in a control system. Brit. J. Psychol., 38:142-148, 1947b.

P. Gawthrop, I. Loram, and M. Lakie. Predictive feedback in human simulated pendulum balancing. Biol. Cybern., 101(2):131-146, 2009.

P. Gawthrop, I. Loram, M. Lakie, and H. Gollee. Intermittent control: a computational theory of human control. Biol Cybern, 104(1-2):31-51, Feb 2011.

P. J. Gawthrop. Physical model-based intermittent predictive control. In T. Kaczorek, editor, Proc. 8th IEEE Int. Conf. Methods \& Models in Automation \& Robotics, pages 707-712, Szczecin, Poland, September 2002.

P. J. Gawthrop. Intermittent constrained predictive control of mechanical systems. In Ian R Petersen, editor, Proc. 3rd IFAC Symposium on Mechatronic Systems, Manly, Australia, 2004.

P. J. Gawthrop. Frequency domain analysis of intermittent control. Proc. I. Mech. Eng. Pt. I: J. Systems Control Eng., 223(5):591-603, 2009.

P. J. Gawthrop and L. Wang. Intermittent predictive control of an inverted pendulum. Control Eng. Pract., 14(11):1347-1356, November 2006.

P. J Gawthrop and L. Wang. Intermittent model predictive control. Proc. I. Mech. Eng. Pt. I: J. Systems Control Eng., 221(7):1007-1018, 2007.

R. Johansson, M. Magnusson, and M. Akesson. Identification of human postural dynamics. IEEE Trans. Biomed. Eng., 35(10):858 - 869, Oct. 1988.

D. L. Kleinman. Optimal control of linear systems with time-delay and observation noise. IEEE Trans. Automatic Control, 14(5):524-527, October 1969.

D. L. Kleinman, S. Baron, and W. H. Levison. An optimal control model of human response. Part I: Theory and validation. Automatica, 6:357-369, May 1970. 
H. Kwakernaak and R. Sivan. Linear Optimal Control Systems. Wiley, New York, 1972.

W. H. Levison, S. Baron, and D. L. Kleinman. A model for human controller remnant. IEEE Trans. ManMachine Systems, 10(4):101-108, Dec. 1969.

D. B. Lockhart and L. H. Ting. Optimal sensorimotor transformations for balance. Nat. Neurosci., 10:1329 $-1336,2007$.

I. D. Loram, H. Gollee, M. Lakie, and P. J. Gawthrop. Human control of an inverted pendulum: Is continuous control necessary? is intermittent control effective? is intermittent control physiological? J Physiol, 589 (2):307-324, 2011.

I. D. Loram, M. Lakie, and P. J. Gawthrop. Visual control of stable and unstable loads: what is the feedback delay and extent of linear time-invariant control? J. Physiol., 587(6):1343-1365, 2009.

K. Masani, A. H. Vette, and M. R. Popovic. Controlling balance during quiet standing: Proportional and derivative controller generates preceding motor command to body sway position observed in experiments. Gait Posture, 23(2):164 - 172, 2006.

C. Maurer and R. J. Peterka. A new interpretation of spontaneous sway measures based on a simple model of human postural control. J. Neurophysiol., 93:189-200, 2005.

D. McRuer. Human dynamics in man-machine systems. Automatica, 16:237-253, May 1980.

F. Navas and L. Stark. Sampling or intermittency in hand control system dynamics. Biophys. J., 8(2):252-302, 1968.

P. D. Neilson. Influence of intermittency and synergy on grasping. Motor Control, 3:280-284, 1999.

P. D. Neilson and M. D. Neilson. A neuroengineering solution to the optimal tracking problem. Hum. Movement Sci., 18(2-3):155 - 183, 1999.

P. D Neilson and M. D. Neilson. An overview of adaptive model theory: solving the problems of redundancy, resources, and nonlinear interactions in human movement control. J. Neural Eng., 2(3):S279-S312, 2005.

P. D. Neilson, M. D. Neilson, and N. J. O'Dwyer. Internal models and intermittency: A theoretical account of human tracking behaviour. Biol. Cybern., 58:101-112, 1988.

J. Nocedal and S. J. Wright. Numerical Optimization. Springer Series in Operations Research. Springer Verlag, 2nd edition, 2006.

Y. Oytam, P. D. Neilson, and N. J. O’Dwyer. Degrees of freedom and motor planning in purposive movement. Hum. Movement Sci., 24(5-6):710 - 730, 2005.

R. J. Peterka. Sensorimotor integration in human postural control. J. Neurophysiol., 88(3):1097-1118, 2002.

R. Pintelon and J. Schoukens. System Identification. A frequency domain approach. IEEE Press, 2001.

R. Pintelon, J. Schoukens, and Y. Rolain. Frequency domain approach to continuous-time identification: some practical aspects. In H. Garnier and L. Wang, editors, Identification of continuous-time models from sampled data, chapter 8, pages 215-248. Springer, 2008.

E. Ronco, T. Arsan, and P. J. Gawthrop. Open-loop intermittent feedback control: Practical continuous-time GPC. IEE Proc. Part D: Control Theory and Applications, 146(5):426-434, September 1999.

H. van der Kooij and E. de Vlugt. Postural responses evoked by platform pertubations are dominated by continuous feedback. J. Neurophysiol., 98:730-743, 2007. 
H. van der Kooij, R. Jacobs, B. Koopman, and H. Grootenboer. A multisensory integration model of human stance control. Biol Cybern, 80(5):299-308, 1999.

H. van der Kooij and R. J. Peterka. Non-linear stimulus-response behavior of the human stance control system is predicted by optimization of a system with sensory and motor noise. J Comput Neurosci, 30 (3):759-778, 2011.

H. van der Kooij, E. van Asseldonk, and F. C. T. van der Helm. Comparison of different methods to identify and quantify balance control. J Neurosci Methods, 145(1-2):175-203, 2005.

M. A. Vince. The intermittency of control movements and the psychological refractory period. Brit. J. Psychol., 38:149-157, 1948.

T. D. J. Welch and L. H. Ting. A feedback model reproduces muscle activity during human postural responses to support-surface translations. J. Neurophysiol., 99:1032-1038, 2008. 


\section{University Library}

\section{- M M I N E R VA A gateway to Melbourne's research publications}

Minerva Access is the Institutional Repository of The University of Melbourne

Author/s:

Gollee, H;Mamma, A;Loram, ID;Gawthrop, PJ

Title:

Frequency-domain identification of the human controller

Date:

2012-09-01

Citation:

Gollee, H., Mamma, A., Loram, I. D. \& Gawthrop, P. J. (2012). Frequency-domain

identification of the human controller. BIOLOGICAL CYBERNETICS, 106 (6-7), pp.359-372. https://doi.org/10.1007/s00422-012-0503-9.

Persistent Link:

http://hdl.handle.net/11343/283035 